\title{
Oscillatory brain activity associates with neuroligin-3 expression and predicts progression free survival in patients with diffuse glioma
}

\author{
Jolanda Derks ${ }^{1,2} \cdot$ Pieter Wesseling $^{2,3,4} \cdot$ Ellen W. S. Carbo $^{1} \cdot$ Arjan Hillebrand $^{5} \cdot$ Edwin van Dellen $^{6,7}$. \\ Philip C. de Witt Hamer ${ }^{8} \cdot$ Martin Klein $^{9} \cdot$ Geert J. Schenk ${ }^{1}$. Jeroen J. G. Geurts ${ }^{1}$. Jaap C. Reijneveld ${ }^{10}$. \\ Linda Douw ${ }^{1,2,11}$
}

Received: 15 May 2018 / Accepted: 1 August 2018 / Published online: 9 August 2018

(c) The Author(s) 2018

\begin{abstract}
Introduction Diffuse gliomas have local and global effects on neurophysiological brain functioning, which are often seen as 'passive' consequences of the tumor. However, seminal preclinical work has shown a prominent role for neuronal activity in glioma growth: mediated by neuroligin-3 (NLGN3), increased neuronal activity causes faster glioma growth. It is unclear whether the same holds true in patients. Here, we investigate whether lower levels of oscillatory brain activity relate to lower NLGN3 expression and predict longer progression free survival (PFS) in diffuse glioma patients.

Methods Twenty-four newly diagnosed patients with diffuse glioma underwent magnetoencephalography and subsequent tumor resection. Oscillatory brain activity was approximated by calculating broadband power $(0.5-48 \mathrm{~Hz})$ of the magnetoencephalography. NLGN3 expression in glioma tissue was semi-quantitatively assessed by immunohistochemistry. Peritumor and global oscillatory brain activity was then compared between different levels of NLGN3 expression with Kruskal-Wallis tests. Cox proportional hazards analyses were performed to estimate the predictive value of oscillatory brain activity for PFS. Results Patients with low expression of NLGN3 had lower levels of global oscillatory brain activity than patients with higher NLGN3 expression $(\mathrm{P}<0.001)$. Moreover, lower peritumor (hazard ratio $2.17, \mathrm{P}=0.008$ ) and global oscillatory brain activity (hazard ratio 2.10, $\mathrm{P}=0.008$ ) predicted longer PFS.

Conclusions Lower levels of peritumor and global oscillatory brain activity are related to lower NLGN3 expression and longer PFS, corroborating preclinical research. This study highlights the important interplay between macroscopically measured brain activity and glioma progression, and may lead to new therapeutic interventions in diffuse glioma patients.
\end{abstract}

Keywords Glioma $\cdot$ NLGN3 $\cdot$ Magnetoencephalography $\cdot$ Neurophysiology $\cdot$ Tumor progression

\section{Introduction}

Gliomas are primary brain tumors originating from (precursors of) glial cells. Most gliomas in adult patients are characterized by diffuse infiltration of tumor cells in the surrounding brain parenchyma. These so-called diffuse gliomas have widespread effects on neurophysiological functioning as measured with magnetoencephalography (MEG) and electroencephalography (EEG): both increased low frequency

Electronic supplementary material The online version of this article (https://doi.org/10.1007/s11060-018-2967-5) contains supplementary material, which is available to authorized users.

Linda Douw

L.douw@vumc.nl

Extended author information available on the last page of the article activity [1,2] and altered synchronization of activity [3-5], which is commonly referred to as functional connectivity, have been reported. Moreover, particularly altered functional connectivity has deleterious correlates: glioma patients with higher delta $(0.5-4 \mathrm{~Hz})$ and/or theta $(4-8 \mathrm{~Hz})$ band connectivity show poorer cognitive performance [6-8], and more often suffer from epileptic seizures $[9,10]$.

Emerging evidence shows that the relationship between altered neurophysiological functioning and glioma is more reciprocal than previously thought. A series of in vivo and in vitro animal experiments show that increased neuronal spiking of neurons surrounding the tumor significantly enhances tumor growth. Neuroligin-3 (NLGN3), a cell adhesion protein on the postsynaptic membrane that is secreted through neuronal activity, is identified as a key contributor to this process [11]. More recently, it is shown that blocking 
Fig. 1 Tumor mask, cortical areas and NLGN3 immunohistochemistry. Example of tumor mask, peritumor and global oscillatory activity regions (a-d): a indicates an exemplar T1 weighted scan (post gadolinium) with low-grade glioma in the left frontal region, $\mathbf{b}$ depicts the manually segmented tumor mask (dark blue) and peritumor area (light blue), c displays the 78 cortical areas used to calculate global oscillatory brain activity in color, and $\mathbf{d}$ contains the atlas regions that fall within the tumor and surrounding tumor area used to calculate peritumor oscillatory brain activity. Examples of tissue expressing neuroligin-3 (e-h): e hippocampus with moderate to strong staining of neuronal cell bodies and their processes, $\mathbf{f}$ low NLGN3 expression outside the cell nuclei, $\mathbf{g}$ moderate NLGN3 expression especially in tumor cell cytoplasm, and $\mathbf{h}$ high NLGN3 expression with strong staining of tumor cells as well as extensive staining of neuropil in between. $A$ anterior, $I$ inferior, $L$ left, $N L G N 3$ neuroligin-3, $P$ posterior, $R$ right, $S$ superior
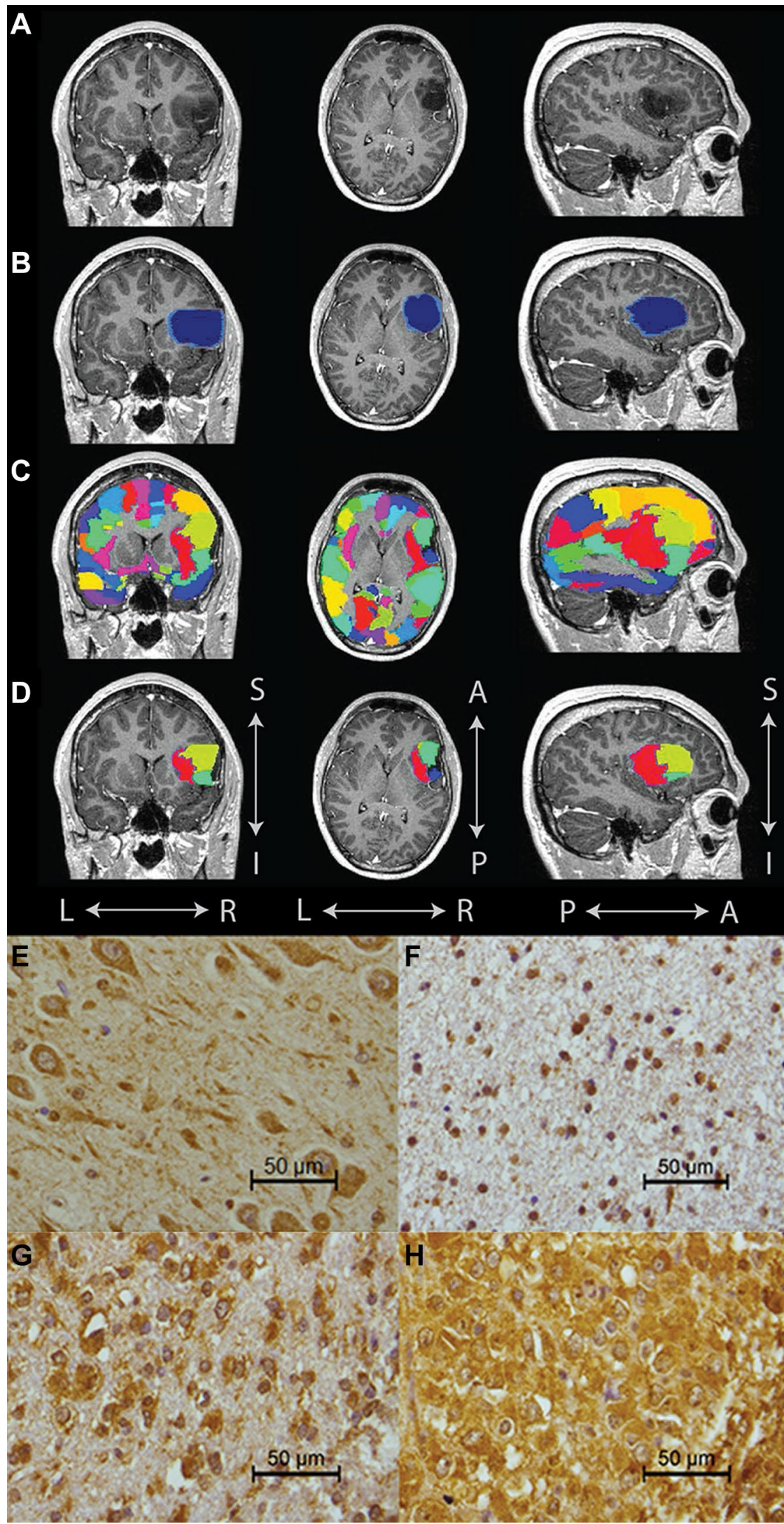
the release of NLGN3 results in diminished tumor growth in animal models of high-grade glioma [12].

The relation between neuronal activity, NLGN3 expression and tumor progression has not been established in a clinical population of patients with diffuse glioma, which hampers clinical exploitation of these obtained fundamental insights. Therefore, we investigated oscillatory brain activity, operationalized as broadband MEG oscillatory power [13]. MEG measures the activity of large populations of neurons oscillating in synchrony and is a non-invasive technique to measure neuronal activity [14]. Moreover, NLGN3 expression was assessed through immunohistochemistry of resected glioma tissue. We hypothesized that patients with lower peritumor and global levels of oscillatory brain activity have lower NLGN3 expression and a longer progression free survival (PFS) than patients with higher levels of oscillatory brain activity.

\section{Methods}

\section{Patients}

Patients visiting the VUmc CCA Brain Tumor Center Amsterdam with suspected de novo diffuse glioma between 2010 and 2012 could participate. Part of this cohort has been described previously $[8,15,16]$. Inclusion criteria were (1) age over 17 years old, (2) histopathologically confirmed World Health Organization (WHO) 2007 grade II, III or IV diffuse glioma [17], (3) at least one seizure (ensuring homogeneity of the cohort in terms of tumor-related epilepsy), and (4) ability to complete neuropsychological testing (data not used in this study). All patients underwent (sub)total tumor resection (as investigated by manual segmentation of remnant tumor tissue using anatomical imaging [LD]), with all patients having at least $76 \%$ of the glioma volume resected. Because of low variation in this cohort, extent of resection was not further explored as a covariate. Patients with prior neurological and psychiatric diseases or previous craniotomies were not eligible for this study.

Tumor progression was based on agreement within the multidisciplinary tumor board of the VUmc CCA Brain Tumor Center Amsterdam taking radiological and clinical information into account. Time to progression was counted from the date of the preoperative MEG in weeks. Patients without progression within the follow-up time frame (June 2017) were censored as of their last contact date.

In addition, 24 sex- and age-matched healthy controls were included to normalize oscillatory brain activity. This study was approved by the ethical review board of the VU University Medical Center, informed consent was obtained from all individual participants included in the study.

\section{Magnetoencephalography}

Oscillatory brain activity was measured non-invasively by MEG [14]. The average time between MEG recording and surgery was 8.4 weeks. Recording and preprocessing methods have been published before $[15,16,18]$ and are explained in detail in the supplementary material. In short, patients underwent a 5-min eyes-closed resting state recording using a 306-channel MEG system (Elekta Neuromag Oy, Helsinki, Finland). Patients' anatomical MRIs were coregistered to the MEG and 78 cortical parcels were selected for analyses $[19,20]$. The MEG time-series were then reconstructed using a scalar beamformer implementation (Elekta Neuromag Oy, version 2.1.28). As a non-invasive measure of neuronal activity, we calculated broadband $(0.5-48 \mathrm{~Hz})$ oscillatory power [13].

\section{Peritumor, global, and non-tumor oscillatory activity}

MR images were used to manually draw the tumor (L.D.; Fig. 1a) to create a MRI tumor mask [21]. Next, the tumor mask was dilated two times with the default FSL kernel (Fig. 1b). The 78 atlas regions were projected onto patients' individual scans (Fig. 1c). The average broadband power of regions overlapping with the dilated tumor mask formed a 'peritumor' oscillatory activity measure per patient (Fig. 1d). Global oscillatory activity was calculated by averaging the absolute power values of all 78 regions per patient. Nontumor oscillatory activity was determined by averaging the absolute power values of all regions except those within the dilated tumor mask. In order to compare these values across patients, individual values for peritumor, global and nontumor oscillatory activity were converted to z-scores using the absolute power values of the entire patient cohort. For global oscillatory power only, z-scores were also calculated based on the values of the healthy controls, in order to allow for comparison of the hazard ratio associated with higher levels of oscillatory activity as compared to healthy levels.

\section{Tissue micro array and immunohistochemistry}

Glioma tissue acquired during surgery (inspected by J.D. and P.W.), was processed in a tissue micro array (TMA) with $0.6 \mathrm{~mm}$ diameter cores and sections were stained for NLGN3 by immunohistochemistry (see supplementary material; Fig. 1e-h). In line with information of the protein atlas (https://www.proteinatlas.org/ENSG000001 96338-NLGN3/tissue), examination of the NLGN3-stained slides revealed abundant expression in neurons. A section of hippocampal tissue was therefore used as positive control (Fig. 1e). Three investigators (J.D., J.J.G.G., G.J.S.) 
semi-quantitatively assessed the NLGN3 staining. For each patient, the core with representative, viable tumor tissue with the highest NLGN3 expression was used for classification of the patients in the low, moderate or high NLGN3 expression group. To classify the cores into low, moderate or high NLGN3 expression, both the intensity and surface area of DAB positivity in the cytoplasm of all cells, as well as DAB in the neuropil were considered (Fig. 1f-h). Some aspects of the samples were not taken into account since they might not accurately represent glioma tissue or NLGN3 expression. These exclusions concerned (1) tissue containing large necrotic areas, (2) tissue without proper quality due to mechanical or technical difficulties, (3) tissue with ample preexistent grey matter (as neuronal cell bodies contain high amounts of NLGN3). Furthermore, staining of the nuclei was ignored because variable and sometimes strong nuclear staining was present in many tumor cores as well as in non-neoplastic tissue samples and may represent nonspecific staining because NLGN3 protein can be expected to be located outside the cell nuclei.

In order to retrospectively assess the isocitrate dehydrogenase $(I D H)$ mutational status of the samples used for this study the TMAs were also immunohistochemically stained for the IDHI R132H (dilution 1:1250, mouse monoclonal, clone H09; dianova GmbH, Hamburg, Germany) mutant protein (a simple, routinely used immunohistochemical tool for detection of about $90 \%$ of all $\mathrm{IDH}$-mutant gliomas).

\section{Possible confounders of oscillatory brain activity and progression free survival}

Several factors may influence oscillatory activity and/or PFS and were included as confounders. For PFS these possible confounders were WHO 2007 tumor grade (grade II or grade III-IV) [17, 22, 23], Karnofsky performance status (KPS) ( $\leq 80$ or $\geq 90$ ) [24], and age (over or under 40 years) [23]. Regarding molecular information, IDHI mutation status is an important prognostic factor and was investigated as a confounder [25]. Moreover, in the case of an $I D H$-mutant glioma, the absence or presence of $1 \mathrm{p} / 19 \mathrm{q}$ codeletion holds additional prognostic information [25]. In a subset of this cohort, $1 \mathrm{p} / 19 \mathrm{q}$ codeletion was determined using loss-of-heterozygosity analysis for clinical purposes. Of note, according to the revised fourth edition of the WHO classification of CNS tumors (published in 2016), diffuse gliomas can be classified in three groups: (1) $I D H$-mutant with $1 \mathrm{p} / 19 \mathrm{q}$ codeletion ('canonical' oligodendrogliomas; most favorable prognosis), (2) IDH-mutant but 1p/19q-non-codeleted (intermediate prognosis), and (3) $I D H$-wildtype (poorest prognosis, often glioblastomas (GBM)) [26, 27]. IDHI-mutant astrocytoma patients without assessment of $1 \mathrm{p} / 19 \mathrm{q}$ status were retrospectively labeled as 1p/19q-non-codeleted. Other confounders that were explored were adjuvant treatment (radiotherapy plus chemotherapy, radiotherapy alone, or no adjuvant treatment), tumor histology (oligodendroglioma, oligoastrocytoma, astrocytoma), midline crossing of the tumor (i.e. bilateral localization) and tumor diameter (diameter $\leq 6 \mathrm{~cm}$ or $>6 \mathrm{~cm}$ in any direction) [23, 24]. We also considered localization in eloquent areas, i.e. areas that if resected would result in loss of function, as a confounder of PFS [24]. Furthermore, oscillatory brain activity may be influenced by age (continuous) [28, 29], tumor volume [1] and patients' head size (based on MRI using SIENA from the FSL toolbox), as larger heads are usually closer to the sensors [14]. Finally, sex was also investigated as a confounder.

\section{Statistical analyses}

Statistical analyses were performed using the PASW Statistics package (version 22.0.0.0, IBM Corp., Armonk, NY, USA) and Matlab (version R2012.a, Mathworks, Natick, MA, USA). Peritumor, global, and non-tumor oscillatory brain activity were normally distributed, tested by Kolmogorov-Smirnov tests $(\mathrm{P}<0.05)$. A Student's $t$ test was used to test differences in global oscillatory brain activity between patients and healthy controls. To test differences in peritumor, global and non-tumor broadband oscillatory brain activity levels between the NLGN3 expression groups, three Kruskal-Wallis tests were performed, each followed by three post hoc Mann-Whitney U pairwise comparisons.

Cox proportional hazards models were used to test whether peritumor, global (z-scores based on the distribution of the patients as well as on the distribution of the control cohort), non-tumor broadband oscillatory brain activity and NLGN3 expression were significant predictors of PFS.

Leave-one-out and permutation analyses were performed to validate the results regarding global broadband oscillatory activity and PFS. For the leave-one-out analyses, 24 Cox proportional hazard models were computed, each time excluding one patient from the entire cohort of 24 patients. For the permutation analyses, global oscillatory brain activity values were randomly shuffled between patients 1000 times, after which P-values for the Cox proportional hazard model were obtained for each permutation separately. From these analyses, a cohort-specific distribution of P-values was created. The experimental P-value was tested against this distribution with a 0.05 alpha cut-off.

Additionally, thirteen multivariate models were created to test the effect of possible confounders. Each model included global broadband oscillatory brain activity with one covariate, due to the small sample size.

A significance level of 0.05 (two-tailed) was used. 


\section{Results}

\section{Patient characteristics}

Twenty-four glioma patients with a mean age of $39 \pm 10.53$ (SD) years were included (Table 1). Sixteen patients had a WHO grade II and six patients a WHO grade III diffuse glioma, while in two patients the diagnosis was GBM (astrocytoma WHO grade IV). In 21 patients, IDHI mutation status was known and for nine patients $1 \mathrm{p} / 19 \mathrm{q}$ codeletion information was available. Five patients had an IDHImutant astrocytoma and were, based on that information, classified as having no $1 \mathrm{p} / 19 \mathrm{q}$ codeletion. This yielded four patients with an $I D H I$-mutant, $1 \mathrm{p} / 19 \mathrm{q}$-codeleted tumor, seven patients with an $I D H I$-mutant, $1 \mathrm{p} / 19 \mathrm{q}$-non-codeleted tumor, and six patients with most likely an $I D H$-wildtype tumor (see Table 1). Seventeen patients showed progression within the follow-up timeframe, with a median PFS of $87 \pm 86$ weeks. Global oscillatory brain activity did not differ between healthy controls and patients $(\mathrm{t}(46)=-0.74$, $\mathrm{P}=0.465)$.

\section{Expression of NLGN3 is associated with oscillatory brain activity}

Expression of NLGN3 could be determined in 21 patients, since tissue of three patients was unavailable. Twelve patients were classified as having low expression of NLGN3, six patients had moderate expression and three patients had high expression.

Peritumor $(\mathrm{H}(2)=9.07, \mathrm{P}=0.011)$, non-tumor $(\mathrm{H}(2)=9.09, \mathrm{P}=0.011)$ and global $(\mathrm{H}(2)=11.13, \mathrm{P}=0.004)$ oscillatory brain activity levels were significantly different according to NLGN3 expression (Fig. 2). Together, these results indicate that not only activity of the tumor region, but also global activity (with and without the tumor included) associates with NLGN3 expression. Post-hoc analyses showed that patients with moderate NLGN3 expression had significantly higher levels of peritumor $(\mathrm{U}=7, \mathrm{P}=0.005)$, global $(\mathrm{U}=2, \mathrm{P}<0.001)$ and non-tumor $(\mathrm{U}=5, \mathrm{P}=0.002)$ oscillatory brain activity than patients with low NLGN3 expression. Levels of peritumor $(\mathrm{U}=0, \mathrm{P}=0.025)$ and global $(\mathrm{U}=1, \mathrm{P}=0.048)$ oscillatory brain activity between patients with high NLGN3 expression and moderate NLGN3 expression were also significantly different: oscillatory activity was lower in the three patients with the highest expression levels.

NLGN3 expression (low and moderate only) was not a predictor of PFS (Hazard Ratio (HR) $0.54,95 \%$ confidence interval $(\mathrm{CI})$ 0.17-1.7, $\mathrm{P}=0.300$ ).
Oscillatory brain activity predicts progression free survival

Univariate Cox proportional hazard models revealed a significant hazard ratio of peritumor (HR 2.17, CI 1.23-3.85, $\mathrm{P}=0.008)$, global (HR 2.10, CI 1.22-3.63, $\mathrm{P}=0.008)$ and non-tumor (HR 2.05, CI 1.18-3.58, $\mathrm{P}=0.011$ ) oscillatory brain activity for PFS, indicating that lower levels of oscillatory brain activity were associated with longer PFS. The result regarding global oscillatory brain activity and PFS was replicated by the leave-one-out analyses, with P-values ranging between 0.004 and 0.036 , and remained significant when creating a sample-specific P-value distribution through permutation analyses. Furthermore, comparable results were found using $\mathrm{z}$-scores based on the mean and SD of healthy controls (HR 1.93, CI 1.19-3.13, $\mathrm{P}=0.008$ ). To visualize this difference in PFS for different levels of global oscillatory brain activity, we applied a median split on the global oscillatory activity values and created Kaplan Meier curves for low and high levels of oscillatory brain activity (Fig. 3, Supplementary Table S1).

The high oscillatory brain activity group included the two GBM patients, which may have skewed these analyses. We therefore repeated the Cox proportional hazard analysis for global oscillatory activity and PFS without the two GBM patients, which yielded comparable results (HR 2.12, CI $1.20-3.79, \mathrm{P}=0.010$ ).

\section{Confounding factors do not influence the predictive value of global oscillatory activity for PFS}

Global oscillatory activity remained a significant predictor of PFS in all analyses when taking several confounders into account (Table 2). Three confounders (almost) reached significance in addition to global oscillatory activity: molecular status was a trend level predictor of PFS (HR 2.33, CI 0.99-5.51, $\mathrm{P}=0.054$ ), tumor histology (HR 2.23, CI 1.03-4.82, $\mathrm{P}=0.041)$ and tumor volume were significant predictors of PFS (HR 1.02, CI 1.00-1.04, P=0.019). Of note, only six patients had a tumor located in an eloquent area, and radiologically none of the patients had a tumor crossing the midline.

\section{Discussion}

Lower peritumor oscillatory brain activity was associated with lower NLGN3 expression and was predictive of longer PFS in a cohort of newly-diagnosed diffuse glioma patients. The predictive value of oscillatory brain activity was valid in the peritumor region, corroborating previous work performed in an animal model of glioma [11]. 


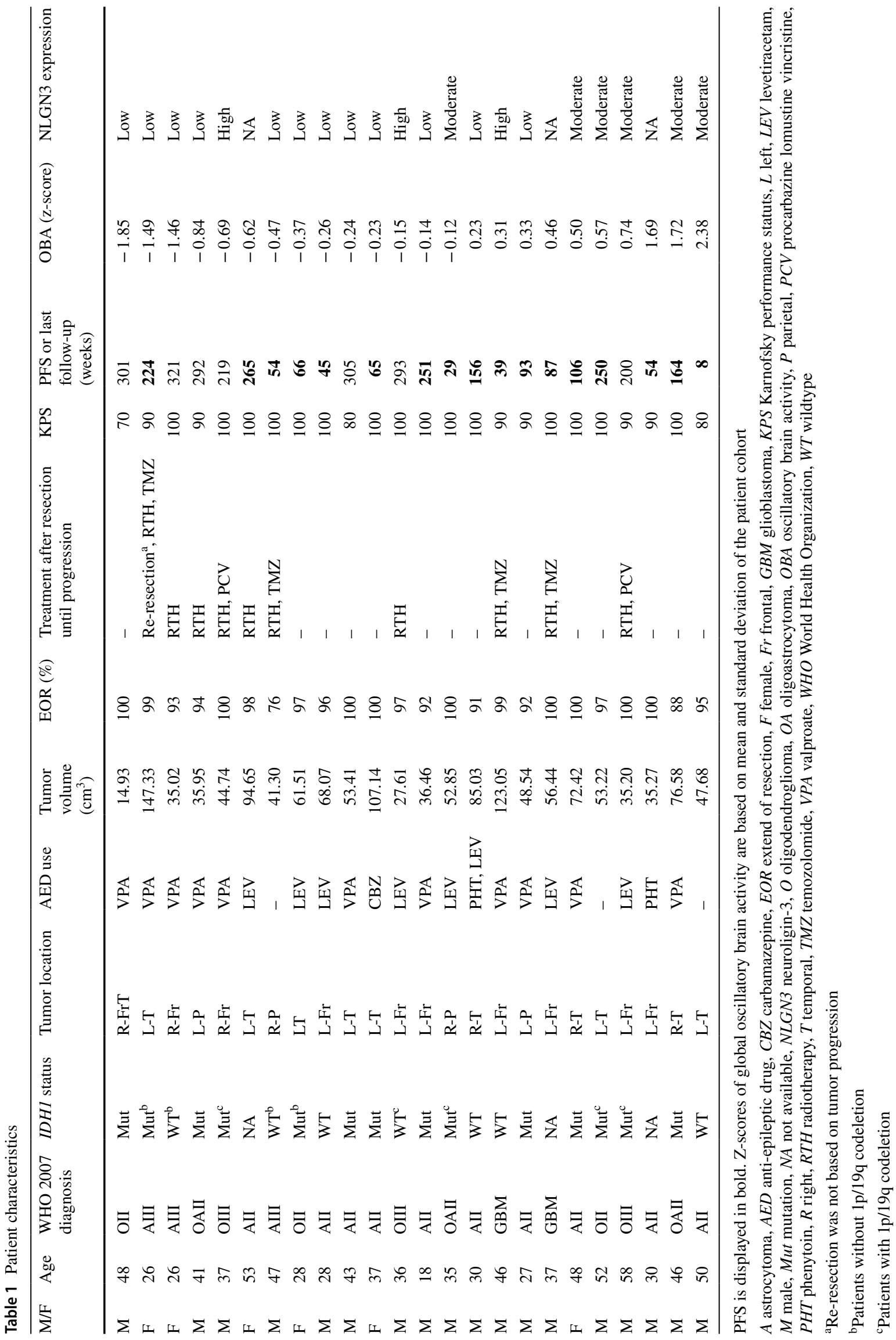




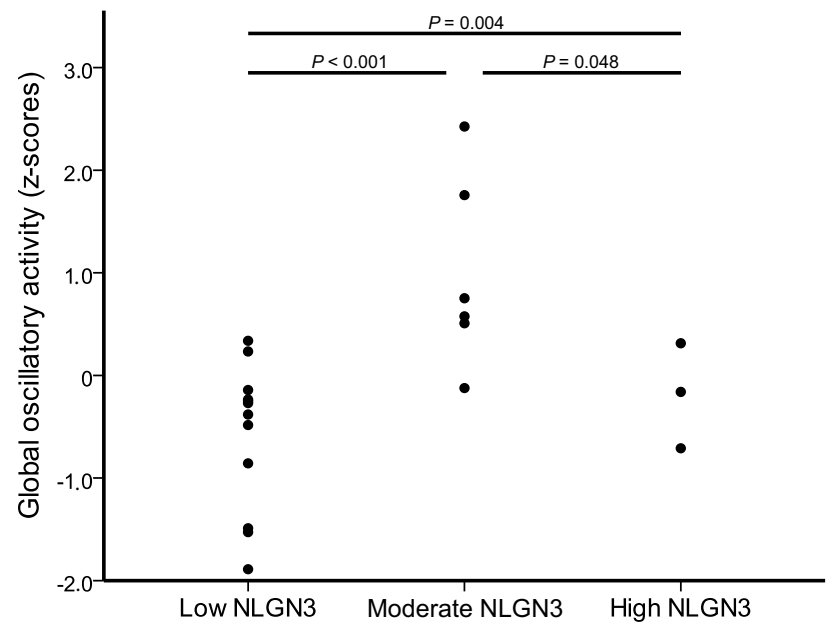

Fig. 2 Z-scores of global oscillatory activity in the three NLGN3 expression groups. Patients with lowest NLGN3 expression showed the lowest global oscillatory activity. NLGN3 neuroligin-3

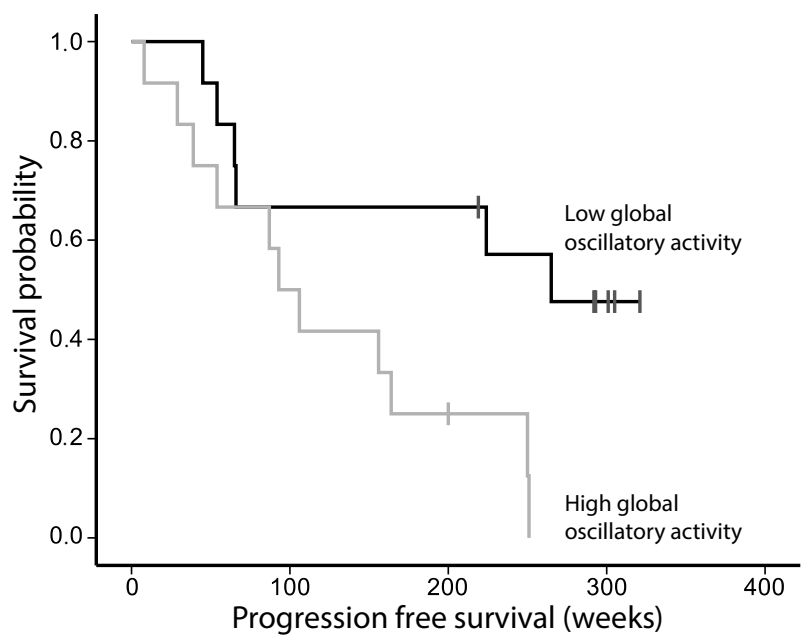

Fig. 3 Kaplan Meier survival curves. Patients with low global oscillatory brain activity $(\mathrm{N}=12$, black line) had longer progression free survival than patients with high global oscillatory brain activity $(\mathrm{N}=12$, grey line), based on a median split of the entire cohort

Moreover, the same results were obtained for activity across the entire brain, extending the current knowledge based on this interesting association between activity, NLGN3 and ultimately survival.

Neuroligin-3 is a tumor growth promoting protein and is secreted through neuronal activity and induces NLGN3 expression in glioma cells [11,12]. NLGN3 is not only secreted through neuronal spiking, but also by activity of oligodendrocyte precursor cells [12,30]. However, only NLGN3 secreted through neuronal spiking has been shown to promote glioma growth [12]. This emphasizes the specificity of brain activity in relation to NLGN3 expression, as corroborated by the association between oscillatory brain activity and NLGN3 expression in our study. As hypothesized, patients with lower expression levels of NLGN3 showed lower levels of oscillatory brain activity than patients with higher expression levels of NLGN3.

Contrary to our expectations, patients with the highest NLGN3 expression levels did not show the highest oscillatory brain activity levels and could be due to sampling issues. Conversely, this could be the result of some sort of inverted U-shape in the association between global oscillatory activity and NLGN3 expression, or of a particular subtype of glioma in this group. Interestingly, two patients with the highest NLGN3 expression had a histological diagnosis of WHO grade III oligodendroglioma, and one can speculate that in these tumors the NLGN3 expression level is relatively high irrespective of the level of NLGN3 that is released by neuronal activity [31]. However, the fact that this group with the highest expression only contains 3 patients precludes drawing firm conclusions for this particular category.

Our results suggest that broadband oscillatory activity may indeed be used as an operationalization of neuronal activity in the context of glioma, for instance to monitor tumor growth. One should keep in mind though that MEG does not measure action potentials directly, but assesses post-synaptic potentials that are indirectly related to neuronal spiking. This means that we cannot be sure that our findings are specific to neuronal spiking. Nevertheless, MEG oscillatory activity remains the most direct non-invasive measure of neuronal activity available at this time [14], and should be further explored as a tool for prognostic purposes.

In addition, our results suggest that (global) brain activity may be a viable treatment target in glioma, possibly in addition to inhibition of NLGN3 secretion [12]. For instance, anti-epileptic drugs diminish neuronal excitability and might therefore slow down tumor growth: several studies have shown survival benefits in GBM patients on anti-epileptic drugs [32-35], although a recent meta-analysis did not confirm this association [36]. Of course, the beneficial effect might be dose dependent [37]. Additionally, the effect may relate to individual differences in the previously unknown and unmeasured levels of neuronal activity, for which we here describe a possible biomarker that may be validated as such in future work.

Of note, the patient cohort in this study does not fully represent the diffuse glioma population in general. This is probably due to the participation bias, patients had to be in a relative good condition in order to be able to undergo MEG and neuropsychological testing. Patients included in our study had a generally high KPS, more frequently a low grade diffuse gliomas, and were relatively young. Another point of discussion is that NLGN3 may well be expressed 
Table 2 Influence of possible confounders

\begin{tabular}{|c|c|c|c|c|c|c|}
\hline \multirow[t]{2}{*}{ Confounders } & \multicolumn{3}{|c|}{ Global oscillatory activity } & \multicolumn{3}{|c|}{ Confounder } \\
\hline & HR & $\mathrm{CI}$ & P-value & HR & CI & P-value \\
\hline Age $(\leq 40)$ & 2.40 & $1.33-4.36$ & $0.004 * *$ & 2.20 & $0.78-3.22$ & 0.138 \\
\hline Age (years continuous) & 2.33 & $1.31-4.13$ & $0.004 * *$ & 0.97 & $0.93-1.02$ & 0.210 \\
\hline Gender & 2.49 & $1.35-4.61$ & $0.004 * *$ & 0.44 & $0.13-1.47$ & 0.183 \\
\hline $\operatorname{KPS}(\leq 80)$ & 2.04 & $1.17-3.58$ & $0.013^{*}$ & 0.49 & $0.06-3.88$ & 0.497 \\
\hline WHO grade & 2.05 & $1.17-3.59$ & $0.013^{*}$ & 1.23 & $0.39-3.93$ & 0.723 \\
\hline Histology & 2.37 & $1.32-4.25$ & $0.004 * *$ & 2.23 & $1.03-4.82$ & $0.041^{*}$ \\
\hline IDH1 mutation status $(\mathrm{N}=18)$ & 2.13 & $1.14-3.97$ & $0.018^{*}$ & 2.10 & $0.66-6.69$ & 0.209 \\
\hline $\begin{array}{l}\text { IDH1 mutation status combined } \\
\text { with } 1 \mathrm{p} / 19 \mathrm{q} \text { codeletion }(\mathrm{N}=18)\end{array}$ & 2.88 & $1.14-7.28$ & $0.025^{*}$ & 2.33 & $0.99-5.51$ & 0.054 \\
\hline Adjuvant treatment & 2.09 & $1.17-3.74$ & $0.013 *$ & 1.02 & $0.52-2.00$ & 0.958 \\
\hline Tumor volume $\left(\mathrm{cm}^{3}\right)$ & 2.29 & $1.33-3.93$ & $0.003 * *$ & 1.02 & $1.00-1.04$ & $0.019 *$ \\
\hline Tumor diameter $(<6 \mathrm{~cm})$ & 2.02 & $1.16-3.51$ & $0.013 *$ & 0.68 & $0.26-1.82$ & 0.445 \\
\hline Non-eloquent area & 2.28 & $1.28-4.06$ & $0.005 * *$ & 0.52 & $0.17-1.60$ & 0.256 \\
\hline Head size & 2.23 & $1.24-4.01$ & $0.007 * *$ & 1.16 & $0.69-1.93$ & 0.580 \\
\hline
\end{tabular}

Results of Cox proportional hazard models for the thirteen separate multivariate analyses using global oscillatory activity combined with each of the confounders

CI 95\% confidence interval, HR hazard ratio, KPS Karnofsky performance status, WHO World Health Organization

$* \mathrm{P}<0.05$; ** $P<0.01$ in a heterogeneous manner in the glioma tissue [38]. Considering the glioma tissue on the TMA, sampling a small area of the tumor might result in inaccurate estimates of expression levels.

In conclusion, lower levels of oscillatory brain activity are associated with lower NLGN3 expression and predict longer PFS. Our study highlights the relevance of neuronal activity for tumor progression in a clinical glioma population, and could be a next step towards improved prognosis and additional treatment strategies.

Acknowledgements The authors would like to thank Nico Akemann, Ndedi Sijsma, Karin Plugge, Marlous van den Hoek, PeterJan Ris for the MEG acquisitions and John Bol for his help with immunohistochemistry.

Funding This study was funded by the Dutch Epilepsy Foundation.

\section{Compliance with ethical standards}

Conflict of interest All authors declare no conflict of interest.

Ethical approval All procedures performed in this study were in accordance with the ethical standards of the institutional research committee and with the 1964 Helsinki declaration and its later amendments or comparable ethical standards. Informed consent was obtained from all individual participants included in the study.

Open Access This article is distributed under the terms of the Creative Commons Attribution 4.0 International License (http://creativeco mmons.org/licenses/by/4.0/), which permits unrestricted use, distribution, and reproduction in any medium, provided you give appropriate credit to the original author(s) and the source, provide a link to the Creative Commons license, and indicate if changes were made.

\section{References}

1. De Jongh A, Baayen JC, De Munck JC et al (2003) The influence of brain tumor treatment on pathological delta activity in MEG. Neuroimage 20:2291-2301. https://doi.org/10.1016/j.neuroimage 2003.07.030

2. Bosma I, Stam CJ, Douw L et al (2008) The influence of lowgrade glioma on resting state oscillatory brain activity: a magnetoencephalography study. J Neurooncol 88:77-85. https://doi. org/10.1007/s11060-008-9535-3

3. Bartolomei F, Bosma I, Klein M et al (2006) How do brain tumors alter functional connectivity? A magnetoencephalography study. Ann Neurol 59:128-138. https://doi.org/10.1002/ana.20710

4. Bartolomei F, Bosma I, Klein M et al (2006) Disturbed functional connectivity in brain tumour patients: evaluation by graph analysis of synchronization matrices. Clin Neurophysiol 117:2039-2049. https://doi.org/10.1016/j.clinph.2006.05.018

5. Guggisberg AG, Honma SM, Findlay AM et al (2008) Mapping functional connectivity in patients with brain lesions. Ann Neurol 63:193-203. https://doi.org/10.1002/ana.21224

6. Bosma I, Douw L, Bartolomei F et al (2008) Synchronized brain activity and neurocognitive function in patients with low-grade glioma: a magnetoencephalography study. Neuro Oncol 10:734744. https://doi.org/10.1215/15228517-2008-034

7. Bosma I, Reijneveld JC, Klein M et al (2009) Disturbed functional brain networks and neurocognitive function in low-grade glioma patients: a graph theoretical analysis of 
resting-state MEG. Nonlinear Biomed Phys 3:9. https://doi. org/10.1186/1753-4631-3-9

8. van Dellen E, Douw L, Hillebrand A et al (2012) MEG network differences between low- and high-grade glioma related to epilepsy and cognition. PLoS ONE 7:e50122. https://doi. org/10.1371/journal.pone.0050122

9. Douw L, van Dellen E, de Groot M et al (2010) Epilepsy is related to theta band brain connectivity and network topology in brain tumor patients. BMC Neurosci 11:103. https://doi. org/10.1186/1471-2202-11-103

10. Derks J, Reijneveld JC, Douw L (2014) Neural network alterations underlie cognitive deficits in brain tumor patients. Curr Opin Oncol 26:627-633. https://doi.org/10.1097/CCO.0000000000 000126

11. Venkatesh HS, Johung TB, Caretti V et al (2015) Neuronal activity promotes glioma growth through neuroligin-3 secretion. Cell 161:803-816. https://doi.org/10.1016/j.cell.2015.04.012

12. Venkatesh HS, Tam LT, Woo PJ et al (2017) Targeting neuronal activity-regulated neuroligin-3 dependency in high-grade glioma. Nature 549:533-537. https://doi.org/10.1038/nature24014

13. Manning JR, Jacobs J, Fried I, Kahana MJ (2009) Broadband shifts in local field potential power spectra are correlated with single-neuron spiking in humans. J Neurosci 29:13613-13620. https://doi.org/10.1523/JNEUROSCI.2041-09.2009

14. Hamalainen M, Hari R, Ilmoniemi RJ et al (1993) Magetoenchepalography-theory, instrumentation, and applications to noninvasive studies of the working human brain. Rev Mod Phys 65(2):413-497. https://doi.org/10.1103/RevModPhys.65.413

15. van Dellen E, Douw L, Hillebrand A et al (2014) Epilepsy surgery outcome and functional network alterations in longitudinal MEG: a minimum spanning tree analysis. Neuroimage 86:354-363. https ://doi.org/10.1016/j.neuroimage.2013.10.010

16. Carbo EWS, Hillebrand A, van Dellen E et al (2017) Dynamic hub load predicts cognitive decline after resective neurosurgery. Sci Rep 7:42117. https://doi.org/10.1038/srep42117

17. Louis DN, Ohgaki H, Wiestler OD et al (2007) The 2007 WHO classification of tumours of the central nervous system. Acta Neuropathol 114:97-109. https://doi.org/10.1007/s00401-007-0243-4

18. van Dellen E, de Witt Hamer PC, Douw L et al (2013) Connectivity in MEG resting-state networks increases after resective surgery for low-grade glioma and correlates with improved cognitive performance. NeuroImage Clin 2:1-7. https://doi.org/10.1016/j. nicl.2012.10.007

19. Tzourio-Mazoyer N, Landeau B, Papathanassiou D et al (2002) Automated anatomical labeling of activations in SPM using a macroscopic anatomical parcellation of the MNI MRI singlesubject brain. Neuroimage 15:273-289. https://doi.org/10.1006/ nimg.2001.0978

20. Gong G, He Y, Concha L et al (2009) Mapping anatomical connectivity patterns of human cerebral cortex using in vivo diffusion tensor imaging tractography. Cereb Cortex 19:524-536. https:// doi.org/10.1093/cercor/bhn102

21. Derks J, Dirkson AR, de Witt Hamer PC et al (2017) Connectomic profile and clinical phenotype in newly diagnosed glioma patients. NeuroImage Clin 14:87-96. https://doi.org/10.1016/j. nicl.2017.01.007

22. Stupp R, Mason WP, van den Bent MJ et al (2005) Radiotherapy plus concomitant and adjuvant temozolomide for glioblastoma. N Engl J Med 352:987-996. https://doi.org/10.1056/NEJMoa0433 30

23. Pignatti F, van den Bent M, Curran D et al (2002) Prognostic factors for survival in adult patients with cerebral low-grade glioma. J Clin Oncol 20:2076-2084. https://doi.org/10.1200/ JCO.2002.08.121

24. Chang EF, Smith JS, Chang SM et al (2008) Preoperative prognostic classification system for hemispheric low-grade gliomas in adults. J Neurosurg 109:817-824. https://doi.org/10.3171/ JNS/2008/109/11/0817

25. Ceccarelli M, Barthel FP, Malta TM et al (2016) Molecular profiling reveals biologically discrete subsets and pathways of progression in diffuse glioma. Cell 164:550-563. https://doi. org/10.1016/j.cell.2015.12.028

26. Louis DN, Perry A, Reifenberger G et al (2016) The 2016 World Health Organization classification of tumors of the central nervous system: a summary. Acta Neuropathol 131:803-820. https://doi. org/10.1007/s00401-016-1545-1

27. Wesseling P, Capper D (2018) WHO 2016 classification of gliomas. Neuropathol Appl Neurobiol 44:139-150. https://doi. org/10.1111/nan.12432

28. Vlahou EL, Thurm F, Kolassa I-T, Schlee W (2014) Resting-state slow wave power, healthy aging and cognitive performance. Sci Rep 4:5101. https://doi.org/10.1038/srep05101

29. Puligheddu M, de Munck JC, Stam CJ et al (2005) Age distribution of MEG spontaneous theta activity in healthy subjects. Brain Topogr 17:165-175. https://doi.org/10.1007/s10548-005-4449-2

30. Zhang Y, Chen K, Sloan SA et al (2014) An RNA-sequencing transcriptome and splicing database of glia, neurons, and vascular cells of the cerebral cortex. J Neurosci 34:11929-11947. https:// doi.org/10.1523/JNEUROSCI.1860-14.2014

31. Kamoun A, Idbaih A, Dehais C et al (2016) Integrated multiomics analysis of oligodendroglial tumours identifies three subgroups of $1 \mathrm{p} / 19 \mathrm{q}$ co-deleted gliomas. Nat Commun 7:11263. https ://doi.org/10.1038/ncomms11263

32. Kerkhof M, Dielemans JCM, van Breemen MS et al (2013) Effect of valproic acid on seizure control and on survival in patients with glioblastoma multiforme. Neuro Oncol 15:961-967. https://doi. org/10.1093/neuonc/not057

33. Barker CA, Bishop AJ, Chang M et al (2013) Valproic acid use during radiation therapy for glioblastoma associated with improved survival. Int J Radiat Oncol Biol Phys 86:504-509. https ://doi.org/10.1016/j.ijrobp.2013.02.012

34. Weller M, Gorlia T, Cairncross JG et al (2011) Prolonged survival with valproic acid use in the EORTC/NCIC temozolomide trial for glioblastoma. Neurology 77:1156-1164. https://doi.org/10.1212/ WNL.0b013e31822f02e1

35. Kim Y-H, Kim T, Joo J-D et al (2015) Survival benefit of levetiracetam in patients treated with concomitant chemoradiotherapy and adjuvant chemotherapy with temozolomide for glioblastoma multiforme. Cancer 121:2926-2932. https://doi.org/10.1002/ cncr.29439

36. Happold C, Gorlia T, Chinot O et al (2016) Does valproic acid or levetiracetam improve survival in glioblastoma? A pooled analysis of prospective clinical trials in newly diagnosed glioblastoma. J Clin Oncol 34:731-739. https://doi.org/10.1200/ JCO.2015.63.6563

37. Redjal N, Reinshagen C, Le A et al (2016) Valproic acid, compared to other antiepileptic drugs, is associated with improved overall and progression-free survival in glioblastoma but worse outcome in grade II/III gliomas treated with temozolomide. J Neurooncol 127:505-514. https://doi.org/10.1007/s11060-016-2054-8

38. Patel AP, Tirosh I, Trombetta JJ et al (2014) Single-cell RNA-seq highlights intratumoral heterogeneity in primary glioblastoma. Science 344:1396-1401. https://doi.org/10.1126/science.1254257 


\section{Affiliations}

\section{Jolanda Derks ${ }^{1,2} \cdot$ Pieter Wesseling $^{2,3,4} \cdot$ Ellen W. S. Carbo $^{1} \cdot$ Arjan Hillebrand $^{5} \cdot$ Edwin van Dellen $^{6,7}$. Philip C. de Witt Hamer ${ }^{8}$. Martin Klein ${ }^{9}$. Geert J. Schenk ${ }^{1}$. Jeroen J. G. Geurts ${ }^{1}$. Jaap C. Reijneveld ${ }^{10}$. Linda Douw ${ }^{1,2,11}$}

1 Department of Anatomy \& Neurosciences, VU University Medical Center, De Boelelaan 1117, 1081 HV Amsterdam, The Netherlands

2 VUmc CCA Brain Tumor Center Amsterdam, De Boelelaan 1117, 1081 HV Amsterdam, The Netherlands

3 Department of Pathology, VU University Medical Center, De Boelelaan 1117, $1081 \mathrm{HV}$ Amsterdam, The Netherlands

4 Department of Pathology, Princess Máxima Center for Pediatric Oncology and University Medical Center Utrecht, Lundlaan 6, 3584 EA Utrecht, The Netherlands

5 Department of Clinical Neurophysiology and MEG Center, VU University Medical Center, De Boelelaan 1117, 1081 HV Amsterdam, The Netherlands

6 Department of Psychiatry, University Medical Center Utrecht, Heidelberglaan 100, 3584 CX Utrecht,

The Netherlands
7 Brain Center Rudolf Magnus, Universiteitsweg 100, 3584 CG Utrecht, The Netherlands

8 Department of Neurosurgery, Neuroscience Campus Amsterdam, VU University Medical Center, De Boelelaan 1117, 1081 HV Amsterdam, The Netherlands

9 Department of Medical Psychology, VU University Medical Center, De Boelelaan 1117, 1081 HV Amsterdam, The Netherlands

10 Department of Neurology, Neuroscience Campus Amsterdam, VU University Medical Center, De Boelelaan 1117, 1081 HV Amsterdam, The Netherlands

11 Athinoula A. Martinos Center for Biomedical Imaging/Massachusetts General Hospital, 149 13th St, Charlestown, MA 02129, USA 